\title{
Sex Chromatin and Nuclear Sexing in Birds ${ }^{1}$
}

\author{
Chaman Lal Arora and M. Dharamarajan ${ }^{2}$ \\ Department of Animal Breeding, Punjab Agricultural University, \\ Hissar (Haryana) India
}

Received July 25, 1969

\section{Introduction}

The determination of sex at an early age is of great importance in poultry especially in hybrids meant for egg production. Retention of males for any length of time will entail unnecessary expenditure on feeding and management. Except for day old testing, using the vent method there is no other criteria for the determination of sex at an early stage. The day old testing requires an extraordinary skill and training. Therefore, it will be desirable to develop a reasonably accurate criteria for sex determination other than day old testing.

The discovery of nuclear satellite in the intermitotic nerve cells of the cat (Barr and Bertram 1949) enabled the distinguishing of sexual dimorphism at the cellular level in interphase cells. This gave incentive to some workers to test its efficacy in deciding the sexual dimorphism in avian species. Some workers have reported that there does exist sexual dimorphism in avian species based on the presence of sex chromatin body where as another group of workers do not agree to this view. Kosin and Ishizaki (1959) reported the presence of sex chromatin body in female Gallus domesticus. Ohno and Kaplan (1960) confirmed the observations and claimed that sex chromatin of the heterogametic hen represents the single Z-chromosome. Ashley and Theiss (1959) could not, however, find sexual dimorphism in domestic fowl and ducks.

The present study was undertaken with a view to test this divergence of view among various workers and to find out its applicability in establishing sexual dimorphism in avian species. The study of sex chromatin provides a wide scope to consider its incidence, position, size and shape. The present study was limited to the observation of the incidence and distribution of sex chromatin body in various tissues of both sexes of three separate species of birds, i.e. Gallus domesticus (domestic fowl); Columba livia (pigeon) and Passer domesticus (House sparrow).

\footnotetext{
1 This study formed part of Dissertation submitted for the Degree of Master of Veterinary Science of Madras University by the senior author.

2 Ex-Professor and Head of the Department of Animal Genetics, Madras Veterinary College.
} 


\section{Material and methods}

Two pairs, each of Gallus domesticus; Columba livia and Passerdomesticus of different ages were taken with a view to study the incidenceof sex chromatin body in three taxonomically different species of birds. Thedifferent tissues taken for study were blood, liver, duodenum and skin. Blood. was taken from the wing vein before killing, and smears were prepared after diluting the blood with an equal amount of isotonic (8.5\%) normal saline on the slide itself. Pieces of liver, duodenum, and skin were taken immediately after killing the birds. The specimens of various tissues were cut into pieces. of not more than $5 \mathrm{~mm}$ thickness and were fixed in Davidson's fluid for 24 . hours. Then they were dehydrated cleared and embedded in paraffin of $57^{\circ} \mathrm{C}$. melting point (Gur 1956). Sections of various thickness i.e. 8 microns, 10 . microns and 16 microns were cut and stained. Sections of 8 micron thickness. were too thin and revealed incomplete nuclei whereas sections of 16 micron. thickness were too thick and revealed overlapping of nuclei and so were unsuitable for cytological studies. Sections of 10 microns proved to be the best for microscopic examination and enabled a clear and precise identification of sex chromatin body. Nuclei were completely discernible and there was. no overlapping.

Staining procedures: The sections were stained by Feulgen method. (Gur 1956) and differential staining (BS-FG) method (Pansegrau and Peterson 1964) with minor modification in timings. The BS-FG method no doubt specific for buccal smears, as reported by the author, was found to be equally good for the tissues in the present study. The blood smears were stained by Leishman stain (Gur 1956) and Giemsa stain (Alfred et al. 1961). In the latter case, the smears after air drying were fixed in Petrunkevitch no. 2 : fixative (Alfred et al. 1961) for 1-2 hours before staining. Four serial sections at random from each tissue of each sex were stained. A total of 80 cell nuclei for studying incidence of sex chromatin were counted in each slide, 20 nuclei being selected at random in alternate serial sections. In each tissue at least five slides were counted at a time. Those possessing chromatin body at the nuclear membrane were only taken as sex chromatin positive and other

Figs. 1-9. 1, photomicrograph of liver of female Gallus domesticus showing sex chromatin. (indicated by arrow mark, Feulgen method). 2, skin of female Gallus domesticus showing sex chromatin (indicated by arrow mark, BS-FG method). 3, duodenum of female Gallus domesticus showing sex chromatin (indicated by arrow mark, Feulgen method). 4, liver of female Columba livia showing the sex chromatin (indicated by arrow mark, BS-FG method). 5, duodenum of female Columba livia showing the sex chromatin (indicated by arrow mark, Feulgen method). 6, skin of female Columba livia showing sex chromatin (indicated by arrow mark, BS-FG method). 7, skin of female Passer domesticus showing sex chromatin (indicated by arrow mark, BS-FG method). 8, duodenum of female Passer domesticus showing sex chromatin (indicated by arrow mark, Feulgen method). 9, liver of female Passer domesticus showing sex chromatin (indicated by arrow mark, Feulgen. method). 


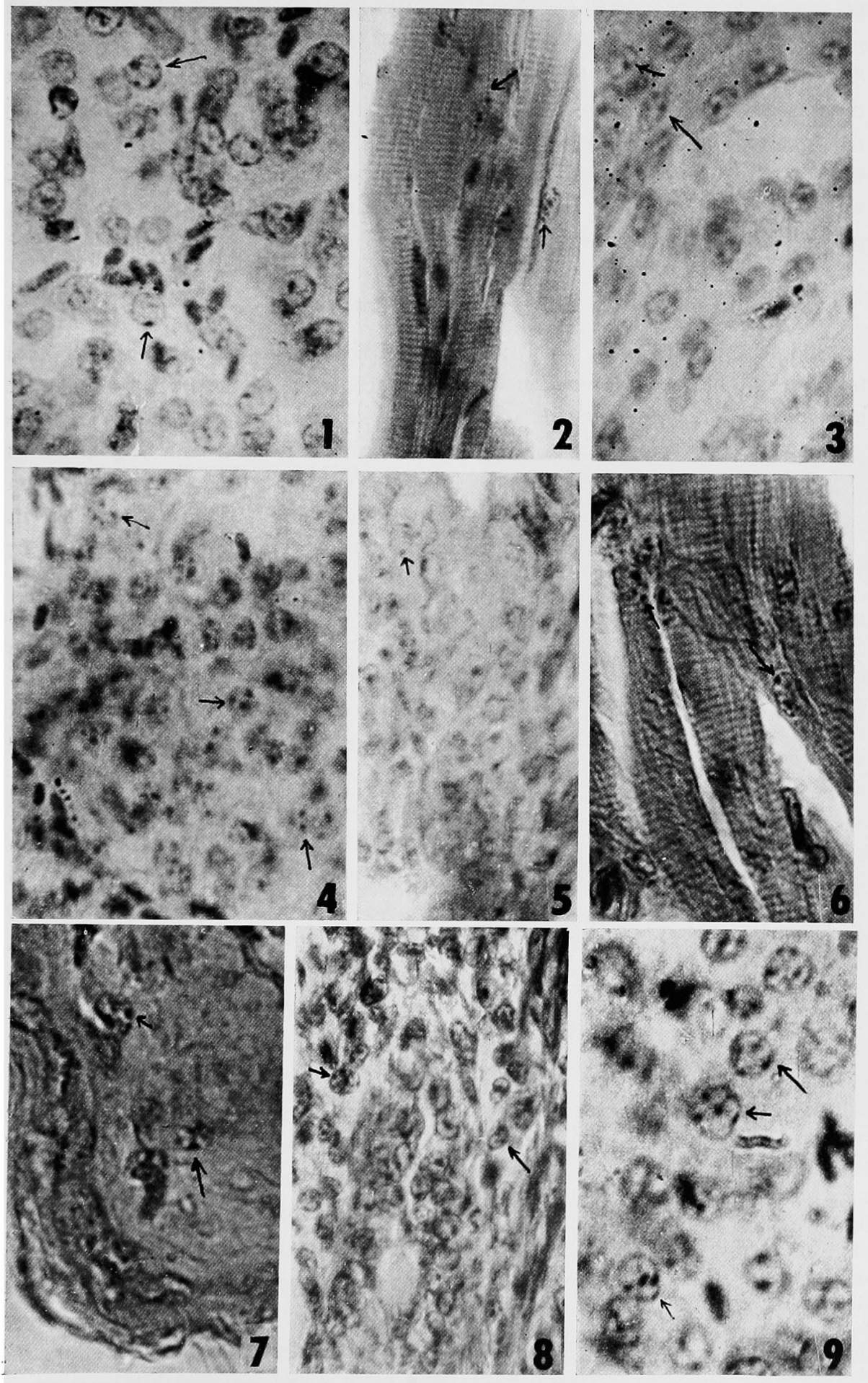


as sex chromatin negative. Over stained, unstained, pycnotic or incomplete nuclei were not included in the count. For drum-sticks and sessile-bodies, 300 polymorphonuclear leucocytes (Heterophils) from each individual at a time were counted. The examination was made with an oil immersion objective giving a magnification of 1200 . A total of 2400 nuclei were counted in each species and in each tissue for incidence of sex chromatin, and 1200 nuclei were counted for the incidence of drumsticks and sessile bodies in polymorphonuclear leucocytes (Heterophils).

\section{Observations}

The sex chromatin was observed in both the sexes in all the tissues taken (Figs. 1-9). The incidence of sex chromatin was more in females than in males. The two methods of staining, i.e. Feulgen and BS-FG methods,
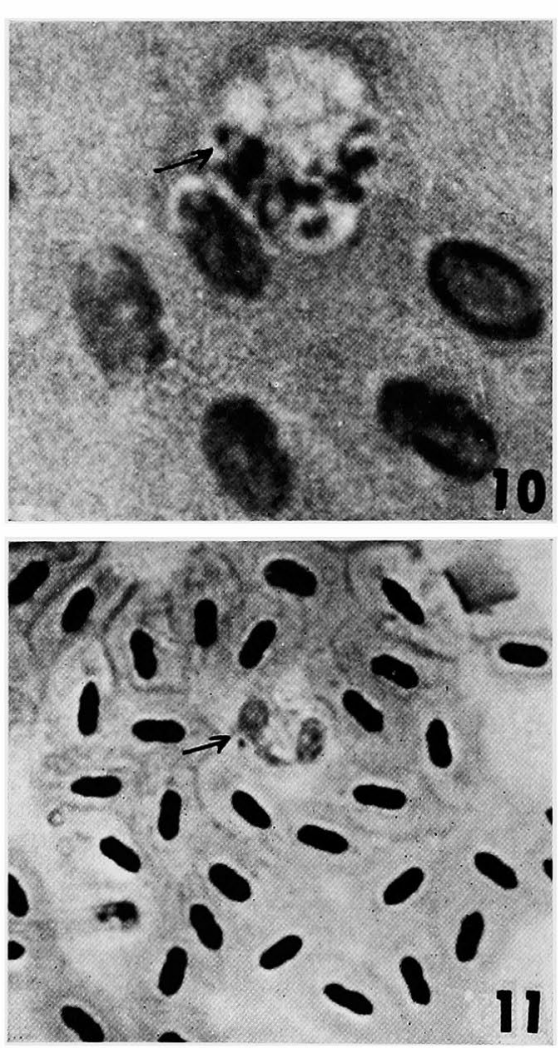

Figs. 10-11. 10, photomicrograph of polymorphonuclear leucocyte (Heterophil) of male Columba livia showing drumstick (indicated by arrow mark, Giemsa stain). 11, polymorphonuclear leucocyte (Heterophil) of male Gallus domesticus showing drumstick (indicated by arrow mark, Giemsa stain). gave similar results, but the latter gave slightly better clarity. The sex chromatin count did not vary much. The male tissues were a bit lightly stained as compared to female tissues in the case of Feulgen method. Incidence of sex chromatin in various tissue in the different species of birds studied are given in Tables 1 and 2 . In some of the cell nuclei more than one sex chromatin clumps were present at the nuclear membrane. In order to avoid error in differentiation, both were considered to be sex chromatin and included in the count. A great care was taken in locating the heteropycnotic body and only those which touched the nuclear wall and had a plano-convex shape were taken in the count. Drum-sticks and sessile bodies in the polymorphonuclear leucocytes were also present in both sexes (Figs. 10-11) but the incidence was more in males than in females. The Giemsa stain in combination with Petrunkevitch fixative no. 2 gave better clarity. The identification and differentiation of drumsticks and sessile bodies from rest of the nuclear lobes of heterophils was better as compared to Leishman stain. The total count got by this method was 
also found to be more than the Leishman method. The results regarding the incidence of drumsticks and sessile bodies are given in Table 3.

Table 1. Incidence of sex chromatin body (Feulgen method)

\begin{tabular}{l|l|c|c|c}
\hline \multirow{2}{*}{ Species } & \multirow{2}{*}{ Sex } & \multicolumn{3}{|c}{ Percentage incidence of sex chromatin body } \\
\cline { 2 - 5 } & & Liver & Duodenum & Skin \\
\hline Gallus domesticus & Male & 20.00 & 26.00 & 24.37 \\
(Domestic fowl) & Female & 54.75 & 52.37 & 50.87 \\
Columba livia & Male & 32.50 & 32.00 & 25.00 \\
(Pigeon) & Female & 57.50 & 56.87 & 42.75 \\
Passer domesticus & Male & 37.00 & 37.37 & 25.62 \\
(House sparrow) & Female & 59.25 & 59.12 & 47.00 \\
\hline
\end{tabular}

Table 2. Incidence of sex chromatin body (BS-FG method)

\begin{tabular}{l|l|c|c|c}
\hline \multirow{2}{*}{ Species } & \multirow{2}{*}{ Sex } & \multicolumn{3}{|c}{ Percentage incidence of sex chromatin body } \\
\cline { 2 - 5 } & & Liver & Duodenum & Skin \\
\hline Gallus domesticus & Male & 25.12 & 25.25 & 24.25 \\
(Domestic fowl) & Female & 47.12 & 46.12 & 43.62 \\
Columba livia & Male & 24.62 & 25.50 & 22.12 \\
(Pigeon) & Female & 48.75 & 44.87 & 47.25 \\
Passer domesticus & Male & 30.62 & 31.87 & 27.75 \\
(House sparrow) & Female & 54.25 & 50.00 & 46.87 \\
\hline
\end{tabular}

Table 3. Incidence of drumsticks and sessile bodies

\begin{tabular}{l|l|c|c}
\hline \multirow{2}{*}{ Species } & \multirow{2}{*}{ Sex } & \multicolumn{2}{|c}{$\begin{array}{c}\text { Percentage incidence of Drumsticks and } \\
\text { sessile bodies }\end{array}$} \\
\cline { 3 - 4 } & & Leishman stain & Giemsa stain \\
\hline Gallus domesticus & Male & 3.34 & 8.66 \\
(Domestic fowl) & Female & 1.41 & 3.99 \\
Columba livia & Male & 3.32 & 12.00 \\
(Pigeon) & Female & 1.00 & 6.46 \\
Passer domesticus & Male & 4.32 & 12.16 \\
(House sparrow) & Female & 1.49 & 5.67 \\
\hline
\end{tabular}

\section{Discussion}

There is divergence of poinion among various workers regarding the existence of sex chromatin body in avian species and the sexual dimorphism for it. Kosin and Ishizaki (1959), Ishizaki and Kosin (1960), Ohno et al. (1960), Moore and Hay (1961) were able to locate sex chromatin in various tisşues of female and male fowl but the existence was more in females than in males, thus establishing a clear sexual dimorphism. Cartoni (1962) taking 
pigeon tissue found incidence of sex chromatin body in female to be $48 \%$ as compared to $14 \%$ in male. Van Limborgh (1964) also observed a sexual dimorphism between male and female chick and duck embryos. Female chick embryos showed the sex chromatin in $49.0-70.5 \%$ of their nuclei as compared to $17.0-26.5 \%$ in males. The corresponding ranges in duck embryos were $51.5-78.0 \%$ in females as against $16.5-28.0 \%$ in males. Katpatal (1961) and Hammer (1964) working with Gallus domesticus and five other species of birds respectively observed a similar incidence of sex chromatin in the two sexes but confirmed existence of sex chromatin in the heterogametic sex in the case of birds. According to the theory of sex chromatin origin, it is derived from one $\mathrm{X}$-chromosome in the case of mammals and it should be present in females which are homogametic but not in males which are heterogametic. In the case of avian species the situation is just reversed because the female is heterogametic and should not exhibit this sex chromatin body. All the above mentioned observations were found to be against the said theory, because all the workers reported the existence of sex chromatin body in the case of female avian species. The present study carried out in three different species of birds, i.e. Gallus domesticus; Columba livia and Passer domesticus, fully confirmed the previous findings of Kosin and Ishizaki (1959), Ishizaki and Kosin (1960), Ohno et al. (1960), Moore and Hay (1961), Cartoni (1962) and Van Limborgh (1964). The difference regarding the percentage of the incidence of sex chromatin in the interphase cell nuclei of different somatic tissues, i.e. liver, duodenal muscle and skin muscle in both females and males, was so great that it can be considered clearly as a creterion for the genetic sex determination. The results were subjected to statistical analysis by applying chi-square test of independence and were found to be significant at both $5 \%$ and $1 \%$ probability levels.

The characteristics of sex chromatin body in the three species do not differ. Ohno et al. (1960) found that single Z-chromosome (like the $\mathrm{X}$-chromosome in mammals) in female birds is positively heteropycnotic in early prophase. It is noted from the present study that sex chromatin in females may not be true replica of single Z-chromosome but only a part of it or it may not necessarily be related to $Z$-chromosome, although it is clearly an incompletely sex limited trait. The study of existence of drumsticks and sessile bodies in polymorphonuclear leucocytes also reveals a clear-cut sexual dimorphism, but their percentage occurrence is quite reverse of the occurrence of sex chromatin. The percentage of drumsticks and sessile bodies is found to be more in males than in females in all the three species of birds here concerned. These findings agreed with the sex chromosome derived drumstick theory and confirmed the previous findings of Beckert (1962) who reported that typical sex chromatin was exclusively found in females in several species of birds but drumsticks were found to be the characteristics of the homogametic sex in birds. In the present study also the incidence of drumsticks and sessile 
bodies was found to be more in males than in females, which further confirms the view of Beckert (1962) that drumsticks and sessile bodies are associated with homogametic sex, whereas sex chromatin is associated with female sex only. The results were analysed statistically and chi-square test was found to be significant at both $5 \%$ and $1 \%$ probability levels. Further it is observed that the nature of sex chromatin and drumsticks was not necessary the same in the case of birds and their properties seemed to be different. This confirmed the earlier findings of Zsifkovits et al. (1959) who conducted a study in rats both before and after castration and reported that the properties of sex chromatin and drumsticks were different.

It can be concluded from the present study that the properties of sex chromatin and drumstick are different and they are differently associated with the two sexes. The sexual dimorphism at the cellular level is so distinct (on the basis of their incidence in two sexes) that it seems reliable to use it as a criterion for determining the genetic sex in the three different species of birds studied.

\section{Summary}

The present study deals with the incidence and distribution of sex chromatin body, drumstick and sessile bodies in two pairs, each of Gallus domesticus, Columba livia and Passer domesticus. Somatic tissues, i.e. liver, duodenum, skin and blood from both sexes were examined. For liver, duodenum and skin interphase nuclei, Feulgen and BS-FG methods were applied and Leishman and Giemsa stains were used for blood. The incidence of sex chromatin was more in females than in males in all the tissues. Incidence of drumsticks and sessible bodies was found to be more in males than in females. The drumsticks were associated with homogametic sex, whereas the sex chromatin body with female sex only. Although the properties of sex chromatin body and drumsticks were not similar and they were differently associated with sex, still the sexual dimorphism at the cellular level was so distinct that it seemed worthwhile to use it as a basis for genetic sex determination in the species of birds studied. The results have been analysed statistically by applying chi-square test with highly significant results.

\section{Acknowledgment}

The authors wish to express their thanks to Dr. R. M. Acharya, Professor and Head, Department of Animal Breeding and Dr. S. N. Kakar, Professor and Head, Department of Genetics, Punjab Agricultural University, Hissar for their valuable suggestions and comments in the preparation of this manuscript. Our thanks are also due to Mr. Lackshman Dass, Stenographer, Department of Genetics, for typing this manuscript. 


\section{Literature cited}

Alfred, M. Lucas and Casimir, Jamroz. 1961. Atlas of Avian Hematology. Agriculture Monograph 25.

Ashley, D. J. B. and Theiss, E. A. 1959. Nuclear sex in species showing male homogametry. Anat. Rec. 135: 115-120.

Barr, M. L. and Bertram, E. G. 1949. A morphological distinction between neuron of the male and female and behaviour of nucleolar satellite during acrenated nucleoprotein synthesis. Nature 163: 676-677.

Beckert, Willium, H. 1964. Sex chromatin in non-mammalian vertebrates. Cited by Cock, A. G. 1964.

Cartoni. 1962. Sex chromatin in the cells of some pigeon tissues. in Anim. Breed. Abst. 32: 93(625). 1964.

Cock, A. G. 1964. Dosage compensation and sex chromatin in non-mammals. Genetical Research 5: 354-363.

Gur, E. 1956. Practical manual of medical and biological staining technique Ed. 1956.

Hammer, 1964. Absence of sex differences in avian chromocentre. Hereditas 51: 191-197.

Ishizaki, H. and Kosin, I. L. 1960. Sex chromatin in early chick embryos. Expt. Cell. Res. 21: 197-200.

Katpatal, B. G. 1961. Sex chromatin in domestic fowl, sheep and goat. Dissertation submitted for the degree of Master of Veterinary Science of Madras University.

Kosin, I. L. and Ishizaki, H. 1959. Incidence of sex chromatin in Gallus domesticus. Science 130: 43-44.

Moore, K. L. and Hay, J. C. 1961. Sexual dimorphism of intermitotic nuclei of birds. Anat. Rec. 139: 315.

Ohno, S., Kaplan, W. D. and Kinosita, R. 1960. On the sex chromatin of Gallus domesticus. Expt. Cell Res. 19: 180-186.

Pansegrau, D. G. and Peterson, R. E. 1964. Improved staining of sex chromatin. Amer. J. Clin. Path. 41: 266-272.

Van Limborgh, J. 1964. The incidence of sex chromatin in the nuclei of amniotic epithelial cell in chick and duck embryos. Acta. morph. mearl. Scand. 5: 366-373.

Zsifkovits, S., Mehes, C. and Jobst, C. 1959. Sex diagnosis by sexual maturation and castration on the sex chromatin pattern in the male rat. Nature 184: 1239-1240. 